\title{
Primary cranial vault germinoma in a 23-year-old man: illustrative case
}

\author{
Christopher Lauren, MD, Donny Argie, MD, Elric B. Malelak, MD, Reza Mawardy, MD, Samuel E. Suranta, MD, Vito M. Junaidy, MD, and \\ Yohanes Firmansyah, MD
}

Department of Neurosurgery, Prof. Dr. W.Z. Johannes Kupang Regional General Hospital, Kupang, East Nusa Tenggara, Indonesia

\begin{abstract}
BACKGROUND Germinoma is the most common type of germ cell tumor that develops intracranially. Germinomas usually grow in the midline structures, such as the pineal and suprasellar regions, and are rarely found in other locations. To the best of the authors' knowledge, no previous research has reported on growth of this tumor in the cranial vault.

OBSERVATIONS The authors reported an unusual case of primary cranial vault germinoma in a young adult. Macroscopically, the tumor had a solidsoft consistency and grayish color with brownish spots on the surface. The histological examination revealed anaplastic cells with round, hyperchromatic, pleomorphic nuclei; prominent nucleoli; and abundant, clear cytoplasm, arranged in lobules and sheets that were infiltrated by lymphocytes and separated by fibrous connective tissue. These findings were consistent with the histopathological characteristics of germinoma.

LESSONS Primary cranial vault germinoma is a unique tumor because no previous research has reported any growth in that location. It should be considered one of the differential diagnoses of lesions located over the cranial vault. Histopathological examination is still the primary modality for diagnosing these tumors and excluding other differential diagnoses.
\end{abstract}

https://thejns.org/doi/abs/10.3171/CASE2082

KEYWORDS germinoma; germ cell tumor; intracranial tumor; neurosurgery

Germinoma is one of the most common types of germ cell tumors that develop in the central nervous system (CNS)..$^{1,2}$ Germinomas account for $3 \%$ to $5 \%$ and $0.4 \%$ to $1 \%$ of intracranial tumors in pediatric and adult populations, respectively.,4 Germinoma is believed to arise from the streaming of primordial germ cells that migrate and are implanted aberrantly in an abnormal location during embryonic development, resulting in transformation into a malignant structure. ${ }^{5,6}$ These tumors usually grow in the pineal and/or suprasellar regions and, rarely, in the basal ganglia, thalamus, cerebral hemispheres, corpus callosum, and posterior fossa. ${ }^{3,7-11}$ To the best of our knowledge, no previous research or literature has reported on growth of this tumor in the cranial vault.

We present a case report of primary cranial vault germinoma that developed in a 23-year-old man. We describe a detailed clinical history, physical examination and neuroradiological findings, operative procedures, histopathological findings, postoperative management, follow-up, and review of the case based on previously reported research and literature.

\section{Illustrative Case \\ History}

A 23-year-old male patient presented to the neurosurgery clinic with a chief concern of chronic headache for the past 5 months. The patient described the headache as a tight band that pressed both sides of his head, was intermittent, developed nearly four times a day, and lasted approximately 30 minutes for each episode. The symptoms worsened and became more frequent 1 month earlier. He regularly took pain relief medication, such as paracetamol and other nonsteroidal anti-inflammatory drugs, for 5 months, but the drugs only temporarily relieved the symptoms. He also reported a small painless bump on the right side of his head that manifested simultaneously with the headaches and had grown slowly since then. No other associated symptoms, such as seizure, weakness, sensory complaints, nausea or vomiting, or visual or endocrine disturbance, were present. The patient did not have any history of similar symptoms, head trauma, or tumor, especially in the brain or spinal region.

ABBREVIATIONS 3D = three-dimensional; $A F P=$ alpha-fetoprotein; $\beta$-hCG $=$ beta-human chorionic gonadotropin; CNS = central nervous system; $C T=$ computed tomography; $\mathrm{MRI}=$ magnetic resonance imaging.

INCLUDE WHEN CITING Published April 5, 2021; DOI: 10.3171/CASE2082.

SUBMITTED October 23, 2020. ACCEPTED January 6, 2021.

(C) 2021 The authors, CC BY-NC-ND 4.0 (http://creativecommons.org/licenses/by-nc-nd/4.0/), 


\section{Examination}

The patient's Karnofsky Performance Scale score was 90 , and his visual analog scale score was 5 . We located a painless bump over the right temporoparietal region approximately $8 \mathrm{~cm}$ in diameter; the height was approximately $0.7 \mathrm{~cm}$ above the skin's surface. The bump had a less defined border. No abnormalities were noted on ophthalmological, endocrinological, or neurological examination.

The patient underwent regular noncontrast computed tomography (CT) scanning, on which the soft-tissue window showed a hyperdense lenticular mass located over the right temporoparietal region. The mass caused shifting of the midline structures $\geq 0.5 \mathrm{~cm}$ to the left, which compressed the right lateral ventricle (Fig. 1A-D). There was also mild dilatation of the left lateral ventricle. The hyperdense mass grew
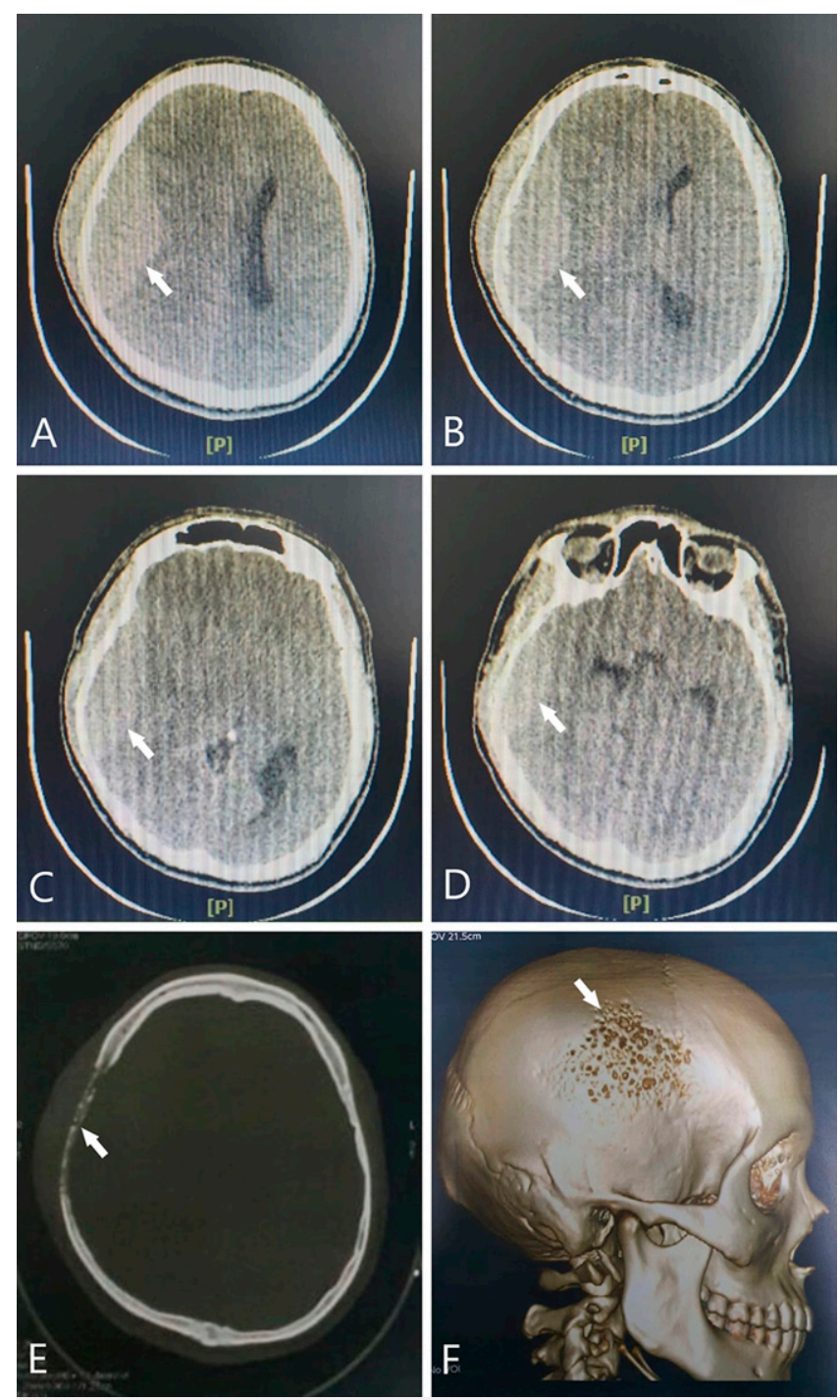

FIG. 1. Noncontrasthead CT scans. A-D: Axial view shows a lenticular mass that grows inside and outside the bone at the right temporoparietal region (white arrows). E: Bone window shows decreased bone density at the right temporoparietal region (white arrow). F: $3 D$ reconstruction shows a lytic lesions of the right temporal and parietal bone (white arrow). outward from the adjacent bone in the right temporoparietal region. No other mass or abnormality was found on other intracranial structures, especially in the pineal and suprasellar regions. The bone window and three-dimensional (3D) reconstructions showed multiple small, round lytic lesions on the right temporal bone and a portion of the right parietal bone (Fig. $1 \mathrm{E}$ and $\mathrm{F}$ ). We could not perform magnetic resonance imaging (MRI) on this patient because no facilities with the necessary equipment exist in East Nusa Tenggara. We chose surgery as the main course of management for this patient to relieve the mass effect and obtain a histopathological analysis of the mass to confirm the diagnosis.

\section{Operative Procedure}

We performed a large skin incision, similar to a trauma flap, on the right side of the patient's head. After the scalp was incised, we encountered a well-defined mass that grew outward from the bone (Fig. 2A). We created a large bone flap around the mass. The mass was growing on the epidural space and adhered to the dura mater below. We performed a gross-total resection of the mass and sent it to the pathology anatomy laboratory for further histopathological examination. The dura mater was also removed with the mass (Fig. 2B). The dural defect was closed with synthetic dura using a watertight closure technique and fibrin glue (Fig. $2 \mathrm{C}$ ). The bone flap was removed together with the mass, and the bone defect was closed with artificial bone made from a polymethyl methacrylate compound and titanium mesh (Fig. 2D). The surgical procedure lasted approximately 3 hours.

\section{Histopathological Features}

The tumor located at the outer side of the bone measured $7 \times 5 \times$ $0.5 \mathrm{~cm}$; the inner tumor measured $10 \times 8 \times 2 \mathrm{~cm}$. Both tumors had a solid-soft consistency and grayish color with brownish spots on the surface (Fig. 3A and B). The microscopic appearance showed a malignant tumor arranged in lobules and sheets separated by fibrous connective tissue and infiltrated by lymphocyte inflammatory cells (Fig. 3C). The tumor was composed of a proliferation of anaplastic cells
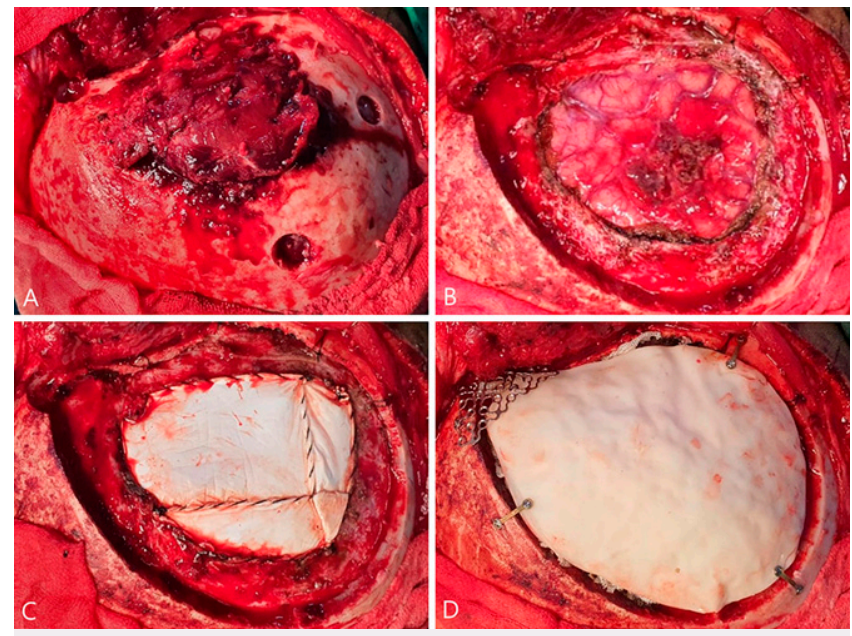

FIG. 2. Surgical procedure. A: Tumor mass growing outward from the bone structure below. B: The mass was resected, together with the bone flap and dura mater. C: The dural defect was closed with synthetic dura. D: The bone defect was closed with bone cement and titanium mesh. 


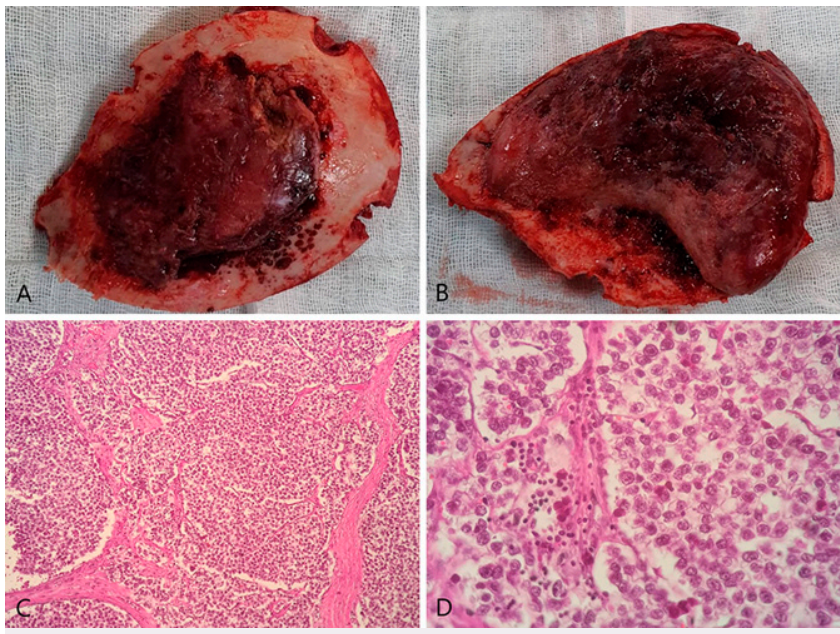

FIG. 3. Macroscopic and microscopic views. A and B: Macroscopic appearance of the mass located on the bone's outer and inner side. C: Microscopic appearance shows the specimen arranged in lobules and sheets separated by a fibrous connective tissue (hematoxylin and eosin, original magnification $\times 100$ ). D: Round, hyperchromatic, pleomorphic nuclei; prominent nucleoli; abundant, clear cytoplasm; apparent mitotic activity; and lymphocytic infiltrates along with the fibrous connective tissue are visible (hematoxylin and eosin, original magnification $\times 400$ ).

with round, hyperchromatic, pleomorphic nuclei; prominent nucleoli; and abundant, clear cytoplasm (Fig. 3D). Mitotic activity was apparent, with the mitotic index measuring 8 to 10 high power fields. The finding was consistent with the characteristics of germinoma. The tissue specimen was examined using hematoxylin and eosin stain and magnifications $\times 100$ and $\times 400$.

\section{Postoperative Management}

Postoperatively, we performed serum and cerebrospinal fluid (CSF) analyses; no tumor cells, beta-human chorionic gonadotropin ( $\beta-h C G)$, or alpha-fetoprotein (AFP) levels were detected on either analysis. No residual tumor or other tumor mass was revealed on the postoperative contrast CT scan of the head and spine. The patient was discharged on postoperative day 5 with no symptoms of headache or neurological deficit. We conducted follow-up with the patient for 5 years; he reported no new or recurrent symptoms and was able to perform daily activities. We could not perform follow-up CT scanning on our patient because he did not have enough money for the procedure and lived on a remote island in East Nusa Tenggara, which prevented him from coming to our hospital. Nearby healthcare facilities did not have CT scanning capability, and we conducted follow-up visits with our patient only by phone.

\section{Discussion}

\section{Observations}

Primary intracranial germinoma is the most frequent type of germ cell tumor involving the CNS.1,2 Germinomas are mostly found in pediatric patients and account for $3 \%$ to $5 \%$ of intracranial tumors in that population, compared with adults, in whom germinomas account for only $0.4 \%$ to $1 \%$ of intracranial tumors. ${ }^{3,4}$ Based on previous research and literature, intracranial germinomas usually grow in the midline structures in descending order: pineal region, suprasellar region, both pineal and suprasellar regions, and, rarely, in the basal ganglia, thalamus, cerebral hemispheres, corpus callosum, and posterior fossae, such as the cerebellum and brainstem. ${ }^{3,7-11}$ Our patient presented a unique case because we found an intracranial germinoma that developed primarily in the cranial vault at the right temporoparietal region, which has not been reported in previous research or literature.

Germinoma is believed to arise from the streaming of primordial germ cells that migrate and are implanted aberrantly in an abnormal location during embryonic development, resulting in a transformation into malignant structures. ${ }^{5,6}$ The process is commonly involved in the midline, but in our patient, it developed far from the midline structures, and the exact mechanism was unknown. Germinoma can spread locally to adjacent brain structure or through the subarachnoid space but is less likely to invade the bone. ${ }^{12}$ There was only one reported primary intrasellar germinoma involving the central skull base structure, which invaded the sphenoid bone and sinus. ${ }^{2}$ In our patient, however, the intracranial germinoma invaded the temporal and parietal bone, which caused the tumor to grow outside the intracranial compartment.

The CT scan of the intracranial germinoma revealed a homogeneous hyperdense lesion compared with the adjacent brain structure and was enhanced with contrast injection. In our patient, we discovered a hyperdense lenticular mass over the temporoparietal region that had an epidural hematoma-like appearance. Other masses that may have a similar appearance include meningiomas, osteomas, hemangiomas, and other metastatic processes. We initially suspected that a disseminating or metastatic germinoma located in the CNS implanted into the epidural space because no cases of germinoma in this location have been reported. However, postoperative contrast-enhanced CT scans of the head and spine confirmed that no other tumor growth source existed. The gold standard imaging modality for germinoma is MRI, but no facilities have the necessary equipment in East Nusa Tenggara, so we performed CT scanning as the first-choice modality for our patient.

Histopathological examination is the main modality for diagnosing germinoma. In our patient, the tumor cells were characterized by round, hyperchromatic, pleomorphic nuclei; prominent nucleoli; and abundant, clear cytoplasm, arranged in lobules and sheets that were infiltrated by lymphocytes and separated by fibrous connective tissue. The findings fit with the characteristics of germinoma, based on the reported literature..$^{1,13}$ Some tumor markers, such as $\beta$-hCG and AFP levels in CSF and serum, are commonly used to detect a germinoma or other germ cell tumor. In a pure germinoma, there are undetectable or normal levels of $\beta$-hCG and AFP in both CSF and serum analyses, as found in our research. 1,2,13-15

The treatment modality for intracranial germinoma consists of surgical management to obtain a tissue biopsy and nonsurgical management, such as radiation therapy and chemotherapy. Radiation therapy remains the primary modality for treating germinoma because of its radiosensitive nature. ${ }^{1}$ Currently, there is an increasing trend toward using a combination therapy of low-dose radiation and chemotherapy because of the fewer side effects compared with radiation therapy alone..$^{1,16}$ In our patient, we performed a gross-total resection of the tumor to relieve the mass effect that occurred and obtain a tissue specimen for histopathological examination. No residual mass was revealed on the postoperative CT scan.

\section{Lessons}

Primary cranial vault germinoma is a unique occurrence because no previous research and literature have reported any growth in that 
location. It should be considered one of the differential diagnoses of lesions located over the cranial vault. Tumor markers and histopathological examination are still the primary modalities for diagnosing these tumors and excluding other differential diagnoses. We encountered some limitations, such as no initial or postoperative MRI data because the necessary facilities do not exist in East Nusa Tenggara, and received minimal data about follow-up imaging because of patient limitations.

\section{References}

1. Osorio DS, Allen JC. Management of CNS germinoma. CNS Oncol. 2015;4(4):273-279.

2. Douglas-Akinwande AC, Mourad AA, Pradhan K, Hattab EM. Primary intracranial germinoma presenting as a central skull base lesion. AJNR Am J Neuroradiol. 2006;27(2):270-273.

3. Villano JL, Propp JM, Porter KR, et al. Malignant pineal germ-cell tumors: an analysis of cases from three tumor registries. Neuro Oncol. 2008;10(2):121-130.

4. McCarthy BJ, Shibui S, Kayama T, et al. Primary CNS germ cell tumors in Japan and the United States: an analysis of 4 tumor registries. Neuro Oncol. 2012;14(9):1194-1200.

5. Fukushima S, Yamashita S, Kobayashi H, et al. Genome-wide methylation profiles in primary intracranial germ cell tumors indicate a primordial germ cell origin for germinomas. Acta Neuropathol. 2017;133(3):445-462.

6. Liang L, Korogi Y, Sugahara T, et al. Dynamic MR imaging of neurohypophyseal germ cell tumors for differential diagnosis of infundibular diseases. Acta Radiol. 2000;41(6):562-566.

7. Goodwin TL, Sainani K, Fisher PG. Incidence patterns of central nervous system germ cell tumors: a SEER Study. J Pediatr Hematol Oncol. 2009;31(8):541-544.

8. Tomura N, Takahashi S, Kato K, et al. Germ cell tumors of the central nervous system originating from non-pineal regions: CT and MR features. Comput Med Imaging Graph. 2000;24(4):269-276.

9. Liang L, Korogi $Y$, Sugahara T, et al. MRI of intracranial germ-cell tumours. Neuroradiology. 2002;44(5):382-388.

10. Wang L, Zhu W, Li X, et al. A rare case report and literature review on primary germinoma in cerebellar hemisphere. Childs Nerv Syst. 2017;33(11):2039-2045.

11. Luo Z, Qian Z, Yang K, et al. Primary germinoma originating from the insular lobe: a case report and review of the literature. World Neurosurg. 2017;98:871.e1-871.e7.
12. Itoyama $\mathrm{Y}$, Kochi M, Yamashiro S, et al. Combination chemotherapy with cisplatin and etoposide for hematogenous spinal metastasis of intracranial germinoma-case report. Neurol Med Chir (Tokyo). 1993;33(1):28-31.

13. Sato K, Takeuchi H, Kubota T. Pathology of intracranial germ cell tumors. Prog Neurol Surg. 2009;23:59-75.

14. Yoshida J, Sugita K, Kobayashi T, et al. Prognosis of intracranial germ cell tumours: effectiveness of chemotherapy with cisplatin and etoposide (CDDP and VP-16). Acta Neurochir (Wien). 1993; 120(3-4):111-117.

15. Calaminus $G$, Bamberg $M$, Harms $D$, et al. AFP/ $\beta$-HCG secreting CNS germ cell tumors: long-term outcome with respect to initial symptoms and primary tumor resection. Results of the cooperative trial MAKEI 89. Neuropediatrics. 2005;36(2):71-77.

16. Calaminus G, Kortmann R, Worch J, et al. SIOP CNS GCT 96: final report of outcome of a prospective, multinational nonrandomized trial for children and adults with intracranial germinoma, comparing craniospinal irradiation alone with chemotherapy followed by focal primary site irradiation for patients with localized disease. Neuro Oncol. 2013;15(6):788-796.

\section{Disclosures}

The authors report no conflict of interest concerning the materials or methods used in this study or the findings specified in this paper.

\section{Author Contributions}

Conception and design: Lauren, Argie, Malelak, Suranta, Junaidy. Acquisition of data: Lauren, Argie, Mawardy, Suranta. Analysis and interpretation of data: Lauren, Argie, Mawardy, Suranta, Firmansyah. Drafting the article: Lauren, Argie, Mawardy, Junaidy. Critically revising the article: Lauren, Malelak, Mawardy, Junaidy. Reviewed submitted version of manuscript: Lauren, Mawardy, Junaidy, Firmansyah. Approved the final version of the manuscript on behalf of all authors: Lauren. Statistical analysis: Lauren, Mawardy, Firmansyah. Administrative/technical/material support: Lauren, Argie, Malelak, Firmansyah. Study supervision: Lauren, Malelak.

\section{Correspondence}

Christopher Lauren: Prof. Dr. W.Z. Johannes Kupang Regional General Hospital, Kupang, East Nusa Tenggara, Indonesia. chrislauren11@ gmail.com. 